The MRCPsych Examination is predominantly for the control of professional standards but has only an indirect effect on the educational experience of trainees. More important has been the establishment of rotational training schemes, which although still with large gaps, ensure a range of experience for the trainee. However, even in the best of these schemes there is frequently a vain hope that teaching is going on more or less automatically whereas it is in fact being neglected.

Educational objectives are an attempt to regulate teaching, both clinical and academic, within the schemes. This is done by setting highly defined objectives for the trainee to attain by the end of a given period of study. Assessment procedures are defined and are carried out at intervals to see that the objectives are being attained. Candidates who fail can then be given the extra tuition they need. In theory this is fine, in practice there are several problems.

Firstly, who sets the objectives? The type of psychiatrist who is specified in a set of objectives tends to be the image of their creator. Researchers demand more research, psychotherapists more sensitivity, and so forth. In fact the ease with which a set of objectives can be drafted is inversely proportional to the number of members of the committee. When disagreement occurs there is a tendency to throw everything in, which is very disagreeable for the traince who has to achieve it all. The problem is intensified when one tries to decide which specialties deserve a place in the scheme. If objectives are not set for a particular subspecialty, then trainees will tend to ignore it.

It is easy to see how a set of objectives, once they have been drafted and agreed, can become a strait-jacket, making future developments harder to achieve. What is the point of discussing objectives when the majority of hospitals do not have the facilities to carry them out? An objective without the means to teach it is an invidious situation for a traince since he is expected to learn what he cannot be taught. Objectives ought to be applied to teachers, but the major criterion by which they could be assessed is their results, which means assessing their trainees. In the situation where facilities and manpower are scarce this system is easily and quickly degraded to one in which regular assess- ment of trainees leads to encouragement or admonishment without corrective action being taken. This situation might also be found when objectives are set for possibly unteachable attributes such as 'original thought', as has been suggested in the past.

These problems arise because of the special characteristics of postgraduate training. Objectives might be useful at ' $A$ ' level or undergraduate level, where there is a fairly clearly defined set of subjects to get through-most of which the learners will have little desire to learn. In professional training, let us hope, the learner's motivation is greater, and instead of channelling it rigidly through a 'system', probably thus stifling it, it should be encouraged to develop in the way most natural to each individual.

The idea of a syllabus for training is one step further on from having objectives. There is a more minute definition of the elements to be achieved or learned. Consequently all the problems defined above are multiplied. In my view a syllabus for the MRCPsych would reduce professional training to schoolwork and is an inappropriate way to help those who find the exam difficult.

The problem then is to ensure minimum standards over the range of subject matter and skills covered by clinical psychiatry, while encouraging the development of each traince in the direction which he himself wishes to go. Perhaps the best way to carry this out is to provide guidelines on basic objectives such as eliciting the mental state. It is to be hoped that the facilities to help a poor traince in these areas would be available in all training schemes; and therefore assessment of the trainees serves a purpose and is appropriate. In the areas of special interest however, I do not feel that any kind of internal assessment is appropriate. No objectives can or should be set for these areas since the trainee who has chosen to put extra work in a special interest is presumably doing his best, and needs guidance rather than motivation. In my view this is the limit to which a set pattern should be imposed upon psychiatric education. In the end training is more a function of the motivation and skills of the teachers than it is about the setting of objectives. Standards will improve when teachers improve.

\title{
Career Development Study
}

The College has commissioned a career development study of Members and Inceptors. Two groups (those who became Members of the Royal College of Psychiatrists between 1975 and 1977 and those who passed the Preliminary Test in 1980 and 1981) will be asked in the course of the next few months to complete questionnaires giving details of their carcers. It is hoped that all who are approached will co-operate in this College-sponsored exercise. It will be carried out by the Medical Care Research Unit of the Department of Community Medicine of Sheffield University, and will be subject to strict standards of confidentiality, with only aggregated data being released or published.

ANDREW Sims Chairman, Research Committee 